This manuscript is hereby submitted for peer-review to the IEEE Transactions on Circuits and Systems I: Fundamental Theory and Applications. The manuscript should be considered as a Transaction Brief.

Manuscript received: (December 2, 2003.)

Affiliation of corresponding author:

Máté Matolcsi

Permanent address:

Alfréd Rényi Institute of Mathematics, Hungarian Academy of Sciences, POB 127 ,

H-1364 Budapest, Hungary

Tel: (+361) 483-8302, Fax: (+361) 483-8333

email: matomate@renyi.hu 


\title{
Minimal positive realizations for a class of transfer functions
}

\author{
Andrea Halmschlager, Máté Matolcsi
}

Andrea Halmschlager is $\mathrm{PhD}$ student at the Mathematics Department of the Technical University, Budapest.

E-mail: andhalm@math.bme.hu

Máté Matolcsi is Research Assistant at the Alfréd Rényi Institute of Mathematics, Budapest 


\begin{abstract}
It is a standard result in linear-system theory that an $n$ th-order rational transfer function of a SISO system always admits a realization of order $n$. In some applications, however, one is restricted to realizations with nonnegative entries (i.e. a positive system), and it is known that this restriction may force the order $N$ of realizations to be strictly larger than $n$. In this brief we present a class of transfer functions where positive realizations of order $n$ do exist. With the help of our restult we give improvements on some earlier results in positive-system theory.
\end{abstract}

\title{
Keywords
}

Positive linear systems, discrete time filtering, minimal realizations

\section{INTRODUCTION}

Let the transfer function

$$
H(z)=\frac{p_{1} z^{n-1}+\ldots+p_{n}}{z^{n}+q_{1} z^{n-1}+\ldots+q_{n}}
$$

of a discrete time-invariant linear SISO system of McMillan degree $n$ be given.

It is a standard result in linear-system theory (see e.g. [7], Chapter 9) that an $n$th order realization of $h(z)$ (i.e. a triple $A \in \mathbb{R}^{n \times n}, b, c \in \mathbb{R}^{n}$ such that $h(z)=c^{T}(z I-A)^{-1} b$ ) always exists. In this note, however, we are interested in the positive realization problem, i.e. finding $A, b, c$ with non-negative entries. The nonnegativity restriction on the entries of $A, b, c$, reflecting physical constraints in applications, may force the dimension $N$ of realizations to be strictly larger than $n$. Such positive systems appear for example in modelling bio-systems, chemical reaction systems, and socio-economic systems, as described in detail in the monograph [7].

Recent applications of positive-system theory include a MOS-based technology for discrete-time filtering (the so-called charge routing networks, see [5]), and the design of fiber optic filters [2]. In fact, the theoretic background in these applications is the following modification of the the positive realization problem: decompose an arbitrary transfer function $H(z)$ as the difference $H(z)=H_{1}(z)-H_{2}(z)$ of two transfer functions with positive realizations of dimension $N_{1}, N_{2}$, respectively, and give a priori estimates on the values of $N_{1}$ and $N_{2}$. This problem will be referred to as the positive decomposition problem. For a more detailed description of how the positive realization problem and the positive decomposition problem are related, see the excellent paper [5] and also Section II below.

It is well-known in positive-system theory that the nonnegativity constraint may force the dimension $N$ of positive realizations to be strictly larger than the McMillan degree $n$ (see [1], [3], [9] for different reasons why this phenomenon may occur). Therefore, the minimality problem (i.e. finding the lowest possible value of $N$ ) is far from trivial, and has been dealt with in a number of papers (see [4], [6], [8], [5]). A general algorithm of determining the lowest possible value of $N$ is not known.

In this note we describe a class of transfer functions where $N=n$ is possible to achieve. The results enable us to give improvements of some estimates in [8] and [5]. 


\section{Minimal REALIZATIONS}

In this section we provide a minimal positive realization for a class of transfer functions, and show how our result can be used to improve dimension estimates in [8] and [5].

Theorem 1: Consider the transfer function

$$
H(z):=\frac{c_{0}}{z-1}+\sum_{j=1}^{n-1} \frac{c_{j}}{z-\lambda_{j}}
$$

where $0 \leq \lambda_{j}<1$ and $c_{j}<0$. The necessary and sufficient condition for $H(z)$ to have a positive realization of dimension $n$ is that $\sum_{j=1}^{n-1}\left(-c_{j}\right) \leq c_{0}$.

Proof. The necessity of the condition is clear, because the first Markov coefficient of $H(z)$ is $h_{1}=$ $c_{0}+\sum_{j=1}^{n-1} c_{j}$, which has to be nonnegative in order to allow the possibility of positive realizations.

The proof of sufficiency is a simple construction of a positive realization $(c, A, b)$. Let $c:=(1,0, \ldots, 0)^{T}$ and $b:=\left(c_{0}+\sum_{j=1}^{n-1} c_{j}, c_{1}\left(\lambda_{1}-1\right), \ldots, c_{n-1}\left(\lambda_{n-1}-1\right)\right)^{T}$ and

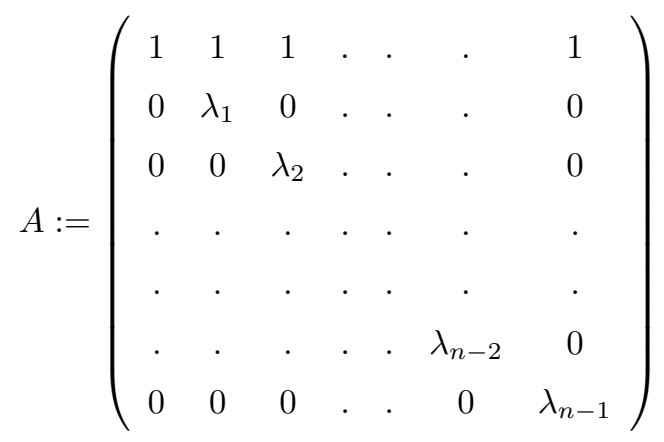

It is straightforward to check that the triple $(c, A, b)$ gives a positive realization of $H(z)$. It is also clear that this realization is minimal, because any realization of $H(z)$ must be of order at least $n$. We remark also, that the dominant pole of $H(z)$ is placed at 1 as a matter of convenience, only; the same construction (with trivial modifications) can be applied, regardless of the position of the (positive) dominant pole, as long as the coefficient of the dominant pole majorizes the sum of the coefficients of the other poles (see Example 1 below).

As a comparison of this theorem with existing results in positive-system theory we note the following:

a; In the case of $n=3$ our result is weaker than that of [6], Theorem 3, where a necessary and sufficient condition is presented for arbitrary signs of $c_{1}, c_{2}$ (whereas we deal with the case $c_{j}<0$ only). It seems, however, that in the case of $n \geq 4$ our result gives a new necessary and sufficient condition for $n=N$ to hold, for a class of transfer functions which has not yet appeared in the literature. We also remark the important fact that our method is constructive, so that a positive realization can be explicitly given.

b; At first glance, the condition $c_{j}<0$ appears to be very restrictive, so that the class of transfer functions considered in Theorem 1 is small. This is not quite the case, however, as demonstrated by the following example. 
Example 1. Consider the transfer function

$$
H(z)=\frac{1}{z-1}-\frac{0.2}{z-0.8}-\frac{0.4}{z-0.7}+\frac{5}{z-0.5}-\frac{0.3}{z-0.4}-\frac{3}{z-0.25}-\frac{2}{z-0.2}
$$

In order to apply Theorem 1 we re-group the terms as

$$
H(z)=\left(\frac{1}{z-1}-\frac{0.2}{z-0.8}-\frac{0.4}{z-0.7}-\frac{0.3}{z-0.4}\right)+\left(\frac{5}{z-0.5}-\frac{3}{z-0.25}-\frac{2}{z-0.2}\right)
$$

We see that in each bracket Theorem 1 can be applied, and therefore $H(z)$ has a positive realization of dimension $4+3=7$.

c; In this and the next point we combine the result of Theorem 1 with existing algorithms in positivesystem theory.

As a corollary of Theorem 1 we can improve the estimates of [8], Theorem 5 (for an illustrative example see Example 2 below).

Corollary 1: Consider the transfer function

$$
H(z)=\frac{1}{z-1}+\sum_{j=1}^{N_{1}} \frac{c_{j}}{z-\lambda_{j}}+\sum_{j=N_{1}+1}^{N_{2}} \frac{c_{j}}{z-\lambda_{j}}+\sum_{j=N_{2}+1}^{N_{3}} \frac{c_{j}}{z-\lambda_{j}}
$$

where $0<c_{j}, 0 \leq \lambda_{j}<1$ for $1 \leq j \leq N_{1}$, and $c_{j}<0,0 \leq \lambda_{j}<1$ for $N_{1}+1 \leq j \leq N_{2}$, and $c_{j} \in \mathbb{R}$, $-1<\lambda_{j}<0$ for $N_{2}+1 \leq j \leq N_{3}$. Assume that $H(z)$ has nonnegative impulse response (this asumption is necessary, but can readily be checked in practice). Then $H(z)$ has a positive realization of dimension not greater than $\tilde{N}+N_{1}+\left(1+N_{2}-N_{1}\right)+2\left(N_{3}-N_{2}\right)$, where $\tilde{N}$ denotes the smallest positive integer such that $\sum_{j=N_{1}+1}^{N_{3}}\left|c_{j}\right|\left|\lambda_{j}\right|^{\tilde{N}} \leq 1$.

Proof. In principle, we follow the idea of the proofs of [8], Theorems 4-5. Let

$$
H_{\tilde{N}}(z):=\frac{1}{z-1}+\sum_{j=1}^{N_{1}} \frac{c_{j} \lambda_{j}^{\tilde{N}}}{z-\lambda_{j}}+\sum_{j=N_{1}+1}^{N_{2}} \frac{c_{j} \lambda_{j}^{\tilde{N}}}{z-\lambda_{j}}+\sum_{j=N_{2}+1}^{N_{3}} \frac{c_{j} \lambda_{j}^{\tilde{N}}}{z-\lambda_{j}}
$$

We can construct a positive realization of $H_{\tilde{N}}(z)$ in $N_{1}+\left(1+N_{2}-N_{1}\right)+2\left(N_{3}-N_{2}\right)$ dimensions in the following way:

The first sum of $N_{1}$ nonnegative poles with positive residues are trivial to realize in $N_{1}$ dimensions in canonical Jordan (diagonal) form.

The second sum containing the nonnegative poles with negative residues can be coupled with the term $\frac{1-\sum_{j=N_{2}+1}^{N_{3}}\left|c_{j} \| \lambda_{j}\right|^{\tilde{N}}}{z-1}$ and then a positive realization of dimension $1+N_{2}-N_{1}$ can be constructed with the help of Theorem 1 (notice that $1-\sum_{j=N_{2}+1}^{N_{3}}\left|c_{j}\right|\left|\lambda_{j}\right|^{\tilde{N}} \geq \sum_{j=N_{1}+1}^{N_{2}}\left|c_{j}\right|\left|\lambda_{j}\right|^{\tilde{N}}$ so that the conditions of Theorem 1 hold). This is where we have an improvement compared to the method of [8].

The third sum containing negative poles can be coupled with the remainder term $\frac{\sum_{j=N_{2}+1}^{N_{3}}\left|c_{j}\right|\left|\lambda_{j}\right|^{\tilde{N}}}{z-1}$ and then the whole sum can be written as

$$
\sum_{j=N_{2}+1}^{N_{3}}\left(\frac{\left|c_{j}\right|\left|\lambda_{j}\right|^{\tilde{N}}}{z-1}+\frac{c_{j} \lambda_{j}^{\tilde{N}}}{z-\lambda_{j}}\right)
$$


Each term in the sum has a two-dimensional positive realization which altogether give a dimension of $2\left(N_{3}-N_{2}\right)$.

After constructing this positive realization of $H_{\tilde{N}}(z)$ we can follow the simple construction of [8], Theorem 5 to get a positive realization of $H(z)$ of dimension $\tilde{N}+N_{1}+\left(1+N_{2}-N_{1}\right)+2\left(N_{3}-N_{2}\right)$.

We give a numerical example to illustrate the result of Corollary 1.

Example 1. Let

$$
H(z)=\frac{1}{z-1}+\frac{8}{z-0.25}-\frac{3}{z-0.4}-\frac{2}{z-0.3}+\frac{5}{z+0.2}
$$

It is easy to see that in this case $\tilde{N}=2$, and

$$
\begin{gathered}
H_{2}(z)=\frac{1}{z-1}+\frac{0.5}{z-0.25}-\frac{0.48}{z-0.4}-\frac{0.18}{z-0.3}+\frac{0.2}{z+0.2}= \\
\left(\frac{0.5}{z-0.25}\right)+\left(\frac{0.8}{z-1}-\frac{0.48}{z-0.4}-\frac{0.18}{z-0.3}\right)+\left(\frac{0.2}{z-1}+\frac{0.2}{z+0.2}\right)
\end{gathered}
$$

We see that $N_{1}=1, N_{2}=3$ and $N_{3}=4$.

The first bracket has a 1-dimensional positive realization:

$c_{1}=1, A_{1}=0.25, b_{1}=0.5$.

We apply Theorem 1 to the second bracket, and get the realization:

$c_{2}=(1,0,0)^{T}$ and $b_{2}=(0.14,0.288,0.126)^{T}$ and

$$
A_{2}=\left(\begin{array}{ccc}
1 & 1 & 1 \\
0 & 0.4 & 0 \\
0 & 0 & 0.3
\end{array}\right)
$$

Then we construct the following 2-dimensional positive realization for the third bracket:

$c_{3}=(0.4,0.16)$ and $b_{3}=(1,0)^{T}$, and

$$
A_{3}=\left(\begin{array}{cc}
0 & 0.2 \\
1 & 0.8
\end{array}\right)
$$

The parallel connection $c=\left(c_{1}, c_{2}, c_{3}\right)$ and $b=\left(b_{1}, b_{2}, b_{3}\right)^{T}$ and

$$
A=\left(\begin{array}{ccc}
A_{1} & 0 & 0 \\
0 & A_{2} & 0 \\
0 & 0 & A_{3}
\end{array}\right)
$$

of these realizations then gives a positive realizaton of $H_{2}(z)$ of dimension 6 . Finally, applying the construction of Theorem 5 in [8] we get the following positive realization of $H(z)$ of dimension 8:

$c_{H}=(0.2,9, \underline{c})$ and $b_{H}=(0,1, \underline{0})$, and

$$
A_{H}=\left(\begin{array}{ccc}
0 & 1 & \underline{0} \\
0 & 0 & \underline{0} \\
\underline{b} & 0 & A
\end{array}\right)
$$


Comparing this with the algorithm described in [8], Theorems 4-5 (which gives a positive realization of $H(z)$ of dimension 11), we can see a considerable improvement. We remark, however, that this general algorithm (as already pointed out in [8]) does not necessarily provide a minimal dimension positive realization of $H(z)$.

d; As a further application of Theorem 1 we give a slight improvement of [5], Theorem 8, concerning the positive decomposition problem. We remark that applications of the positive decomposition problem in the design of charge routing networks and fiber optic filters are given in [5] and [2].

Corollary 2: Let $H(z)$ be a strictly proper asymptotically stable transfer function of order $n$ with simple (possibly complex) poles. Then $H(z)$ can be realized as the difference of an $N$ dimensional positive system and a 1-dimensional positive system with

$$
N=1+n+N_{2}+\sum_{i \geq 3}(i-2) N_{i}
$$

where $N_{2}$ is the number of negative real poles of $H(z), N_{3}$ is the number of pairs of complex poles of $H(z)$ belonging to $\mathcal{P}_{3}$, and $N_{i}$ is the number of pairs of complex poles belonging to

$$
\mathcal{P}_{i} \backslash \cup_{j=3}^{i-1} \mathcal{P}_{j}
$$

(where the region $\mathcal{P}_{j}$ denotes the set of points in the complex plane that lie in the interior of the regular polygon with $j$ edges having one vertex in point 1 and center at $0 . \mathcal{P}_{j}$ can be defined in polar coordinates as in $[5]$ :

$$
\mathcal{P}_{j}:=\left\{(\rho, \theta): \rho \cos \left[\frac{(2 k+1) \pi}{j}-\theta\right]<\cos \frac{\pi}{j}\right\}
$$

with $k=0,1, \ldots, j-1$.) The term $1+n$ in the formulation of $N$ can be reduced to $n$ if $H(z)$ does not contain nonnegative poles with negative residue.

Proof. Note that the improvement compared to [5] is that in our formulation the number $N_{2}$ does not include the number of nonnegative poles with negative residues. In case the number of such poles is 0 or 1 we do not have an improvement. However, if the number of such poles is at least 2, our dimension $N$ is strictly less than that of [5], Theorem 8.

To prove Corollary 2 we apply the constructions of the proof of [5], Theorem 8, with approprite modifications when dealing with nonnegative poles with negative residues.

Write $H(z)=H_{1}(z)-H_{2}(z)$, where $H_{2}(z):=\frac{R}{z-1}$ with $R>0$ sufficiently large. Then $H_{2}(z)$ is a one-dimensional positive system, therefore it is enough to construct a positive realization of $H_{1}(z)$ of the prescribed dimension $N$.

Consider the real poles of $H_{1}(z)$ first.

A partial fraction part of $H_{1}(z)$ of the form $\frac{c}{z-\lambda}$ has a positive realization of dimension 1 if $c>0, \lambda>0$.

A partial fraction part of $H_{1}(z)$ of the form $\frac{R_{j}}{z-1}+\frac{c_{j}}{z-\lambda_{j}}$ (with $R_{j}>0$ sufficiently large), has a positive realization of dimension 2 if $\lambda_{j}<0$. (This is the point of our improvement: we do not need to include the case $\lambda_{j}>0$ and $c_{j}<0$ here, but we treat them separately in the next step.) 
With the help of Theorem 1 we can handle the nonnegative poles with negative residues all together, as opposed to the one-by-one method of the original proof. Indeed, a partial fraction part of $H_{1}(z)$ of the form $\frac{R_{K}}{z-1}+\sum_{j=1}^{K} \frac{c_{j}}{z-\lambda_{j}}$ (with $R_{j}>0$ sufficiently large, $\lambda_{j} \geq 0$ and $c_{j}<0$ ), has a positive realization of dimension $K+1$ by Theorem 1 .

Finally, the complex poles can be treated as in [5]: a partial fraction part of $H_{1}(z)$ of the form $\frac{R_{j}}{z-1}+\frac{c_{1, j}+c_{2, j} z}{\left(z-\lambda_{j}\right)\left(z-\bar{\lambda}_{j}\right)}$ (with $R_{j}>0$ sufficiently large), has a positive realization of dimension $j$ if $\lambda \in \mathcal{P}_{j}$. (For the non-trivial proof of this result we refer to [5].) A parallel connection of these realizations gives a positive realization of $H_{1}(z)$ of dimension $N$.

We also see from the construction that if the number $K$ of nonnegative poles with negative residues is 0 , then the " +1 " dimension in the formula above is not needed, and if $K=1$, then we get the same dimension as in [5], Theorem 8.

\section{Conclusion}

In the course of this brief we constructed a minimal positive realization for a class of transfer functions $H(z)$. Combining our result with existing algorithms we gave improvements of some constructions in positive-system theory. The combination of the ideas resulted in improved dimension estimates for positive realizations. 


\section{REFERENCES}

[1] B.D.O. Anderson, New developments in the theory of positive systems in: Byrnes, Datta, Gilliam, Martin, (Eds.) Systems and Control in the Twenty-First Century, Birkhäuser, Boston 1997.

[2] L. Benvenuti and L. Farina, "The design of fiber-optic filters", Journal of Lightwave Technology, vol. 19, pp. 1366-1375, 2001.

[3] L. Benvenuti, L. Farina, "An example of how positivity may force realizations of 'large' dimensions," Syst. Contr. Lett., vol. 36, pp. 261-266, 1999.

[4] L. Benvenuti and L. Farina, A note on minimality of positive realizations, IEEE Trans. Circuits Syst. I, vol. 45, pp. 676-677, June 1998.

[5] L. Benvenuti, L. Farina, and B.D.O. Anderson, "Filtering through a combination of positive filters," IEEE Trans. Circuits Syst. I, vol. 46, pp. 1431-1440, Dec. 1999.

[6] L. Benvenuti, L. Farina, B. D. O. Anderson and F. De Bruyne, Minimal positive realizations of transfer functions with positive real poles, IEEE Trans. Circuits Syst. I, vol. 47, pp. 1370-1377, Sept. 2000.

[7] L. Farina and S. Rinaldi, Positive linear systems: Theory and applications. New York: Wiley, 2000.

[8] C. Hadjicostis, "Bounds on the size of minimal nonnegative realizations for discrete-time LTI systems," Syst. Contr. Lett., vol. 37, pp 39-43, 1999.

[9] B. Nagy, M. Matolcsi, "A lowerbound on the dimension of positive realizations," IEEE Trans. Circuits Syst. I, vol. 50, pp. 782-784, June, 2003. 